\title{
Characterization of Materials by Raman Scattering
}

\author{
M. KOZIELSKI* \\ Faculty of Technical Physics, Poznań University of Technology \\ Piotrowo 3, 60-965 Poznań, Poland
}

(Received October 27, 2006)

\begin{abstract}
The paper reports on the use of phonon spectra obtained with the Raman spectroscopy for characterization of different materials. The Raman scattering spectra obtained for zinc selenide crystals, mixed crystals zinc selenide admixtured with magnesium or beryllium, oxide crystals including strontium lanthanum gallate, molecular crystals of triammonium hydrogen diseleniate and a homologous series of polyoxyethylene glycols are analysed.
\end{abstract}

PACS numbers: 78.30.- j, 62.20.--x, 61.72.--y, 36.20.Ng

\section{Experimental}

Zinc selenide (ZnSe) and mixed crystals $\left(\mathrm{Zn}_{1-x} \mathrm{Mg}_{x} \mathrm{Se}\right.$ and $\left.\mathrm{Zn}_{1-x} \mathrm{Be}_{x} \mathrm{Se}\right)$ were obtained by the high-pressure Bridgman method. A thermal treatment of ZnSe crystals in liquid zinc was applied to change the mobility and concentration of free charge carriers and to remove structural defects. The two-phase crystals of zinc selenide type $\mathrm{Zn}_{1-x} \mathrm{~A}_{x}$ Se were inhomogeneous crystals with the composition gradient towards the direction of the crystal growth. The composition gradient was a result of segregation of $\mathrm{Mg}$ or Be atoms on the crystal growth.

The crystals of strontium lanthanum gallate $\left(\mathrm{SrLaGaO}_{4}-\mathrm{SLG}\right)$ were obtained by the Czochralski method. Their quality depended on the direction of the crystal growth, temperature gradient on the border of the melt and the crystal, purity of the initial materials, and the composition of the atmosphere of the crystal growth. The SLG crystals grew in the directions $\langle 100\rangle,\langle 001\rangle$, and $\langle 110\rangle$.

The crystals of triammonium hydrogen diseleniate $\left(\left(\mathrm{NH}_{4}\right)_{3} \mathrm{H}\left(\mathrm{SeO}_{4}\right)_{2}-\right.$ TAHSe) were obtained from water solutions. They belong to the group of molecular crystals with hydrogen bonds. They are characterised by proton conductivity and show the ferroelectric phase transition.

*e-mail: markoz@phys.put.poznan.pl 
The polyoxyethylene glycols chosen for the study were those representing the homologue series of $\mathrm{HO}\left(\mathrm{CH}_{2} \mathrm{CH}_{2} \mathrm{O}\right)_{n} \mathrm{H}$ (PEG). These polymers of the molecular weight up to 1000 are liquids at room temperature, while those of the molecular weight above 1000 are solids. They are widely used in industry as surfactants, densifying agents, stabilizers or wetting agents.

\section{Raman scattering in crystals}

\subsection{Plasmon-photon interaction}

In the crystals from groups II-VI, free charge carriers interact with the longitudinal lattice vibrations through macroscopic electric fields. As a result, instead of undisturbed longitudinal optical modes, the Raman spectra show mixed plasmonphonon modes $[1,2]$. Raman scattering related to the plasmon-phonon interaction can be explained in terms of fluctuation of the charge density $\left(I_{\mathrm{B}}\right)$ or the mechanism of the deformation potential and the electrooptical mechanism $\left(I_{\mathrm{A}}\right)$. The interaction of the free charge carriers caused by these two mechanisms is governed by the same selection rules as the interaction of a plasmon with a longitudinal optical phonon. The resultant intensity of scattering is given by $[1,3]$ :

$$
I=I_{\mathrm{A}}+I_{\mathrm{B}} \approx(A+B)\left(\omega_{r} / c\right)^{2}\left(n_{\omega}+1\right) \operatorname{Im}(-1 / \varepsilon) .
$$

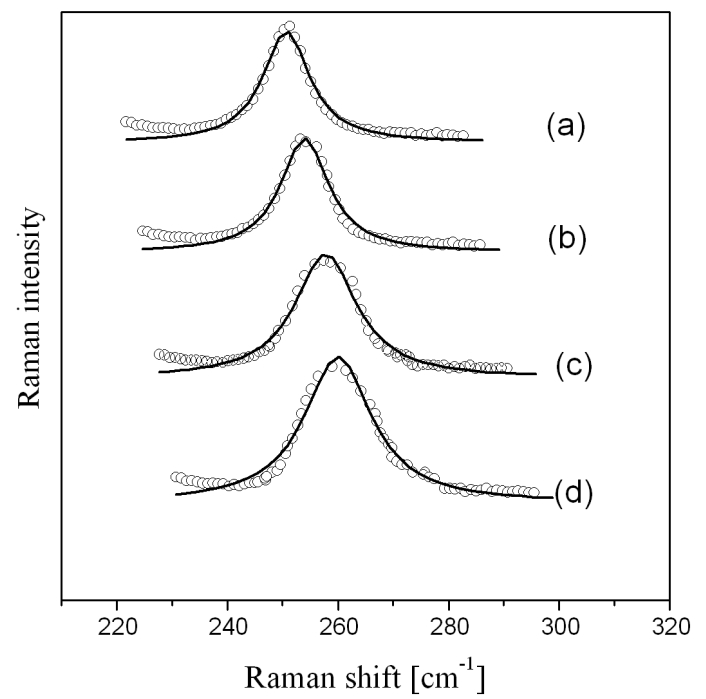

Fig. 1. The Raman spectra of ZnSe crystals with different free carrier concentrations. Empty points - experimental data, solid line - theoretical fit, $n$ - concentration, $\omega_{\mathrm{p}}$ - plasmon frequency, $\gamma$ - plasmon damping constants are the fitting parameters. (a) $n=0.7 \times 10^{17} \mathrm{~cm}^{-3}, \quad \omega_{\mathrm{p}}=69.7 \mathrm{~cm}^{-1}, \gamma=210.1 \mathrm{~cm}^{-1} ;$ (b) $n=$ $1.9 \times 10^{17} \mathrm{~cm}^{-3}, \omega_{\mathrm{p}}=124.5 \mathrm{~cm}^{-1}, \gamma=343.5 \mathrm{~cm}^{-1}$; (c) $n=3.0 \times 10^{17} \mathrm{~cm}^{-3}, \omega_{\mathrm{p}}=$ $150.0 \mathrm{~cm}^{-1}, \gamma=258.3 \mathrm{~cm}^{-1}$; (d) $n=5.9 \times 10^{17} \mathrm{~cm}^{-3}, \omega_{\mathrm{p}}=207.8 \mathrm{~cm}^{-1}, \gamma=$ $311.7 \mathrm{~cm}^{-1}$. 
The constants $A$ and $B$ depend on the concentration of free charge carriers, frequency of the longitudinal optical phonon (LO), transversal optical phonon (TO) and plasmon, the damping constants of plasmon and phonon; $n_{\omega}$ is the Bose-Einstein factor, while $\omega_{r}$ is the frequency of the scattered phonon.

For ZnSe crystals the position and FWHM of the TO band do not depend on the concentration of charge carriers, while the position and FWHM of the LO band do $[4,5]$. Figure 1 presents results of the theoretical fit obtained by the method of the differential transversal scattering cross-section (solid line) to the experimental LO band (points). The parameters of the fit were the plasmon frequency $\omega_{\mathrm{p}}$, plasmon damping constant $\gamma$ and free charge carriers' concentration $n$ [6]. The plasmon damping constant determines the contribution of $I_{\mathrm{A}}$ and $I_{\mathrm{B}}$ to the intensity of Raman scattering. For ZnSe crystals the plasmon frequency $\omega_{\mathrm{p}}$ has always been lower than the plasmon damping constant $\gamma$. This means that in these crystals plasmon has been totally damped, so the deformation potential and electrooptical mechanisms are mainly responsible for the free carriers scattering [5].

The free charge carrier concentrations calculated on the basis of the Raman scattering spectra (Fig. 1) are compared with the corresponding values determined on the basis of the Hall effect measurements (Fig. 2). The results obtained by the two methods are in agreement.

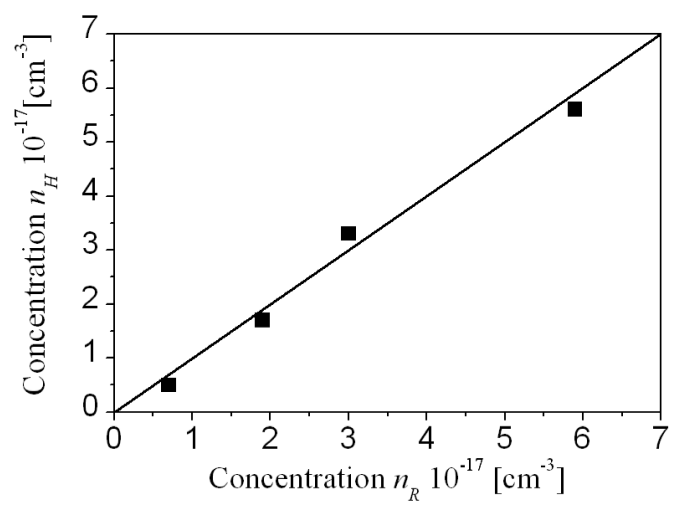

Fig. 2. The free carrier concentration in the ZnSe crystals obtained from the Hall measurements $\left(n_{\mathrm{H}}\right)$ versus the free carrier concentration calculated from the Raman $\operatorname{spectra}\left(n_{\mathrm{R}}\right)$.

As follows from the above, on the basis of the Raman spectra recorded for $\mathrm{ZnSe}$ crystals it is possible to determine the concentration of free charge carrier concentration and the mechanism of their scattering. As this method is non-destructive, it can be applied for investigation of ready elements.

\subsection{Elasticity coefficients}

In a uniformly deformed crystal the character of the interatomic bonds changes. The knowledge of the changes permits describing the elastic properties of 
the crystal. The theoretical model describing the changes relates the interaction forces between atoms with the elasticity constants of the crystal [7-9]. For the crystals of the regular structure $(\mathrm{ZnSe})$, the model should take into account the repulsion forces dependent on the effective charge of ions distributed in the crystal lattice. The elasticity coefficients $C_{i j}$ and the internal stress parameter $\zeta$ are then expressed in terms of the central force constant proportional to the force stretching the bond $(\alpha)$, non-central force constant proportional to the force bending the bond $(\beta)$, and the force constant responsible for the Coulomb interaction $(S)$. For the regular structure these coefficients are [9]:

$$
\begin{aligned}
& C_{11}=(\alpha+3 \beta) a^{-1}-0.0829 S C_{0}, \\
& C_{12}=(\alpha-\beta) a^{-1}-0.136 S C_{0}, \\
& C_{44}=(\alpha+\beta) a^{-1}-0.136 S C_{0}-C \zeta^{2}, \\
& C=(\alpha+\beta) a^{-1}-0.266 S C_{0}, \\
& \zeta=\left[(\alpha-\beta) a^{-1}-0.294 S C_{0}\right](1 / C) .
\end{aligned}
$$

In the above formulae $C_{0}$ has a dimension of the elasticity constant, $a$ is the lattice constant of the regular structure, $V_{a}$ is the elementary cell volume, $\mu$ is the reduced mass. On the basis of measurements of LO and TO bands positions the parameter $S$ was determined from

$$
S=\left(V_{a} \mu / 4 \pi \mathrm{e}^{2}\right)\left(\omega_{\mathrm{LO}}^{2}-\omega_{\mathrm{TO}}^{2}\right) .
$$

Of particular importance is the possibility of determination of the internal stress parameter, whose direct measurement, e.g. by X-ray method is very difficult for most materials.
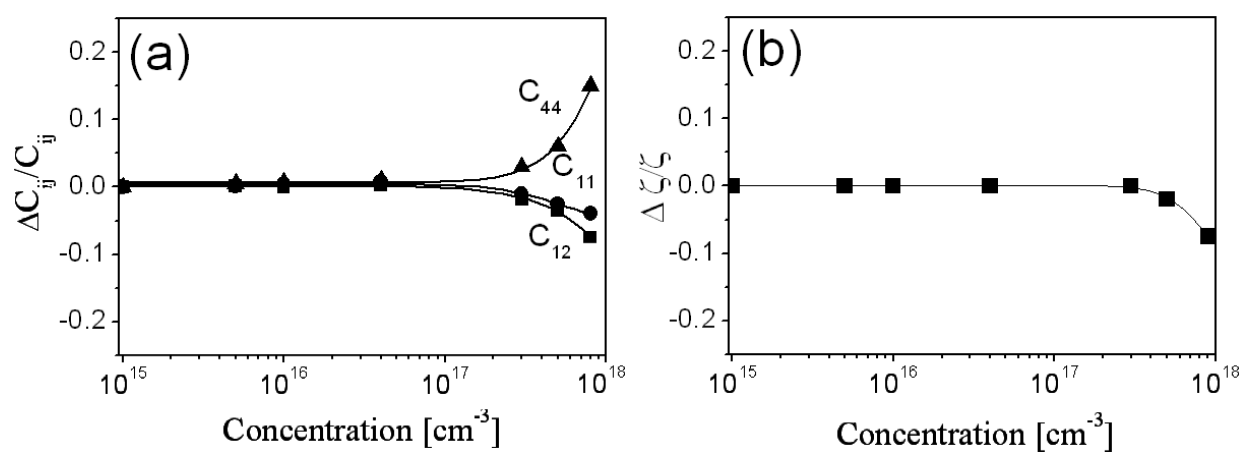

Fig. 3. Relative changes in the elastic constants $C_{i j}$ (a) and internal parameter $\zeta$ of ZnSe crystals (b) as a function of the free carrier concentration. Solid points experimental data, solid lines — obtained assuming the theory proposed by Martin [9].

Figure 3 presents a relative change in the elasticity coefficients $C_{i j}$ and a relative change in the parameter of the internal stress $\zeta$ for $\mathrm{ZnSe}$ crystals as a function of the concentration of free charge carriers. In ZnSe crystals obtained by 
the high-pressure method of Bridgman, there are always regions with the structural defects: excess of selenium and twinnings. The crystal annealing in liquid zinc changes the concentration of free charge carriers and removes the structural defects thus reducing the internal stress in the crystal.

\section{Raman scattering in diphase crystals}

\subsection{Localised modes}

Analysis of the diphase crystals $\mathrm{A}_{1-x} \mathrm{~B}_{x} \mathrm{C}$ as pseudo-diatomic ones can be performed within the model of a linear diatomic chain. The model permits determination of the frequency of localised modes. The replacement of one atom by another in the crystal lattice results in the appearance of a local mode positioned above the branch of optical modes and/or an internal mode localised between the optical and acoustic branches $[10,11]$. The determination of the position of the mode assigned to the newly introduced atom B gives information on the behaviour of optical phonons in the crystals. ZnSe can be one- or two-mode crystals.

Local modes in the crystals can be easily measured, but the internal modes can be observed only in low temperatures at which their damping is very low. The mode damping depends on the density of states so the internal modes can be observed for the crystals for which the separation of the LO-TO modes is large. Moreover, the internal mode must have a frequency close to that of the optical phonon.

Raman scattering spectra of the crystals $\mathrm{Zn}_{1-x} \mathrm{Mg}_{x}$ Se and $\mathrm{Zn}_{1-x} \mathrm{Be}_{x} \mathrm{Se}$ are composed of two separated pairs of phonon bands, Fig. $4[12,13]$. One pair of optical phonons are $\mathrm{TO}(\mathrm{ZnSe})$-like and $\mathrm{LO}(\mathrm{ZnSe})$-like, while the other TO(MgSe)-like and LO(MgSe)-like for the crystals ZnMgSe or TO(BeSe)-like and $\mathrm{LO}(\mathrm{BeSe})$-like for the crystals ZnBeSe. A comparison of the Raman scattering spectra of the two crystals reveals a different position of the TO and LO bands assigned to the MgSe and BeSe crystals. This is related to the presence of the local mode of different frequencies corresponding to the introduced $\mathrm{Mg}$ or Be atoms.

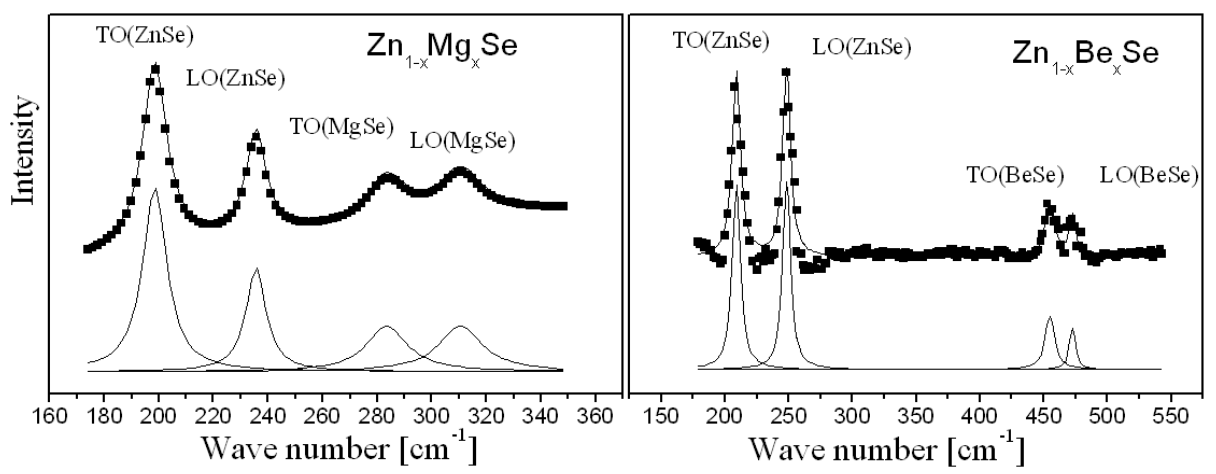

Fig. 4. The Raman spectra of $\mathrm{Zn}_{1-x} \mathrm{Mg}_{x} \mathrm{Se}(x=0.34)$ and $\mathrm{Zn}_{1-x} \mathrm{Be}_{x} \mathrm{Se}(x=0.17)$ crystals. Solid points - experimental data, solid lines - the numerical fit. 


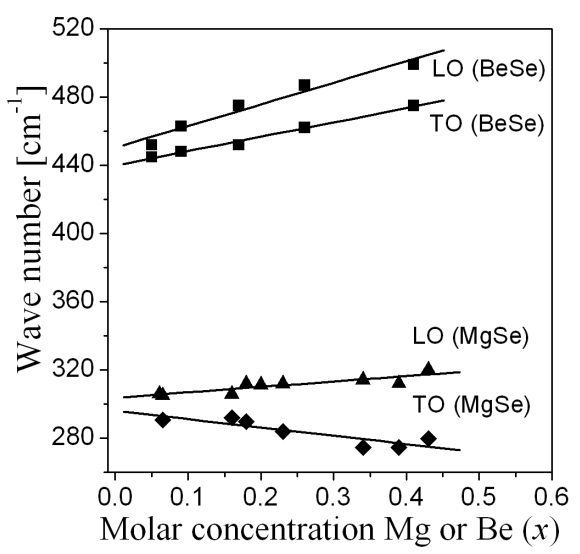

Fig. 5. The dependence of MgSe-like and BeSe-like TO and LO modes on the molar concentration $\mathrm{Mg}$ or Be. Solid points - experimental data, solid lines - curves calculated assuming the modified random element isodisplacement (MREI) model.

The replacement of the $\mathrm{Zn}$ atom in the ZnSe crystal with Be or Mg results in the appearance of local modes above the optical branch.

An increase in the molar concentration due to the introduction of the new atom into the diphase crystal results in an increase in the separation of the optical phonons $\mathrm{TO}(\mathrm{MgSe})-$ like and $\mathrm{LO}(\mathrm{MgSe})-$ like, and $\mathrm{TO}(\mathrm{BeSe})-$ like and $\mathrm{LO}(\mathrm{BeSe})-$ -like, and simultaneously a decrease in the separation of the modes $\mathrm{TO}(\mathrm{ZnSe})$ -like and $\mathrm{LO}(\mathrm{ZnSe})-$ like. As a consequence, the modes coming from $\mathrm{ZnSe}$ are degenerated to the internal mode. The values of the separation of the optical TO-like and LO-like modes characterise the interatomic bonds $\mathrm{Zn}-\mathrm{Se}, \mathrm{Mg}-\mathrm{Se}$ and Be-Se. Figure 5 presents the separation of the optical phonons LO and TO for the crystals ZnMgSe and ZnBeSe and their linear fit. The assumed linear character of the changes in the positions of the optical phonon bands as a function of the molar concentration of the atom introduced permitted drawing the equations for the frequency of the local modes at the limiting value of the concentration $(x=0)$. Therefore, it is possible to predict the behaviour of the optical phonons for higher concentrations of the atom introduced and to determine the frequencies of the modes for the limiting concentration $(x=1)$, that is to determine the positions of the optical phonons for the MgSe and BeSe crystals.

\subsection{Material constants}

The diphase crystals of the type $\mathrm{A}_{1-x} \mathrm{~B}_{x} \mathrm{C}$ can be also analysed in terms of the MREI model [14], defined by macroscopic parameters of the $\mathrm{AC}$ and $\mathrm{BC}$ crystals. The model describes the behaviour of optical phonons in diphase crystals as a function of the molar concentration of the atom introduced $x$. The crystal is analysed for two limiting concentrations of the atom introduced: $x=0$ corresponds to the $\mathrm{AC}$ crystal, while $x=1$ to the $\mathrm{BC}$ crystal. For the limiting case $x=0$, the frequency of the longitudinal mode LO is $\omega_{\text {LOAC }}$, while the frequency of the 
transversal mode TO is $\omega_{\text {TOAC }}$, so the frequencies of the AC crystal modes. The other modes in the Raman scattering spectra are the local modes of the admixture. Analogously, when $x=1$, the frequency of the longitudinal mode LO is $\omega_{\mathrm{LOBC}}$, while that of the TO mode is $\omega_{\mathrm{TOBC}}$, it means the frequencies of the modes of the BC crystals. The other LO and TO modes are the internal modes of the atom introduced. If the frequency of the phonon coming from the atom introduced is higher than that of the phonons of the parent crystal ZnSe, the mode is called local. When the frequency is in the gap between those of the optical and acoustic modes then the mode is called internal.

The MREI model assumes that the force constants depend on the constants of the crystal lattice of the crystals studied and can be approximated by a linear function [15]. As a result of the theoretical calculations for the limits of concentration of the atom introduced $(x=0$ and $x=1)$, the frequencies of the admixture modes are

$$
\begin{aligned}
& \omega_{\mathrm{loc}}=\left[\frac{F_{\mathrm{BC}}+F_{\mathrm{AB}}}{m_{\mathrm{C}}}\left(1+\Theta \frac{a_{\mathrm{BC}}-a_{x}}{a_{\mathrm{BC}}}\right)\right]^{1 / 2}, \\
& \omega_{\mathrm{int}}=\left(\frac{F_{\mathrm{AC}}+F_{\mathrm{AB}}}{m_{\mathrm{B}}}\right)^{1 / 2},
\end{aligned}
$$

where $a_{\mathrm{BC}}$ - the lattice constant of the BC crystal, $a_{x}$ - the lattice constant with the $x$ content dopant, $F_{\mathrm{AC}}, F_{\mathrm{BC}}$, and $F_{\mathrm{AB}}$ are the force constants of the interactions between the atoms of the $\mathrm{AC}, \mathrm{BC}$, and $\mathrm{AB}$ crystals, respectively.

Assuming the MREI model, changes in the optical phonon modes were determined for both crystals ( $\mathrm{ZnMgSe}$ and $\mathrm{ZnBeSe}$ ) in the whole range of molar fraction of the atom introduced, and not only for the molar concentrations used in the experiment. Figure 6 presents changes in the optical phonon positions as a function of the molar concentration of $\mathrm{Mg}$ and $\mathrm{Be}$ (points) and the results of the fit within the MREI model (solid lines). Analysis of the width of the LO-TO

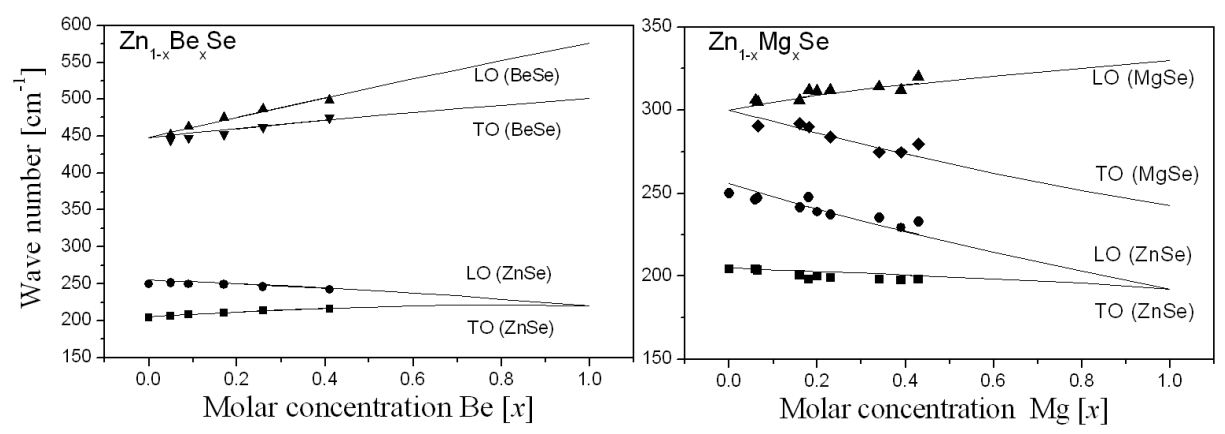

Fig. 6. The frequencies of optical phonons in $\mathrm{Zn}_{1-x} \mathrm{Mg}_{x}$ Se and $\mathrm{Zn}_{1-x} \mathrm{Be}_{x}$ Se crystals at room temperature for different molar concentrations of $\mathrm{Mg}$ or Be. Solid points experimental data, solid lines - the fit by the MREI model. 
phonons split for the limit concentration values $(x=0$ and $x=1)$ has shown that their largest separation occurs for the MgSe crystal, lower for BeSe, and the lowest for $\mathrm{ZnSe}$, which means that the bond strength between the atoms in these crystals must have changed.

On the basis of the fit to the experimental results, assuming the MREI model, the force constants of the interactions between the particular atoms have been found. The force constants were also calculated for the hypothetical crystals MgSe and BeSe, for which certain theoretical parameters were determined. Analysis of the force constant values obtained characterising the interactions between $\mathrm{Zn}-\mathrm{Se}$, $\mathrm{Mg}-\mathrm{Se}, \mathrm{Be}-\mathrm{Se}, \mathrm{Zn}-\mathrm{Mg}$, and $\mathrm{Zn}-\mathrm{Be}$, showed that the highest value characterised the interactions between $\mathrm{Zn}-\mathrm{Se}$, the lower ones - between Be-Se and $\mathrm{Mg}-\mathrm{Se}$. Because of the strong interaction between the $\mathrm{Zn}-\mathrm{Se}$ atoms the separation in the optical phonons in the ZnSe spectrum is low. The smallest separation between the LO-TO phonons obtained for the MgSe crystal (corresponding to the lowest force constant of the interactions between $\mathrm{Mg}-\mathrm{Se}$ ) testifies to an increase in the ionic character of the bond.

\section{Raman scattering in oxide crystals}

To get thin film superconducting materials of satisfactory quality the crystal structure of their supports must be very close to that of the superconductor. The crystal structure of the support must be devoid of macroscopic defects and its lattice constants must match those of the film. It has been shown that the oxide crystals $\mathrm{SrLaGaO}_{4}$ of the perovskite structure make a very good support material for thin film superconducting ceramics. The hitherto studies have shown the formation of point defects during the SLG crystal growth. The defects cause local changes in the state of the charge leading to formation of dye centres and thus to the crystal colouration [16].
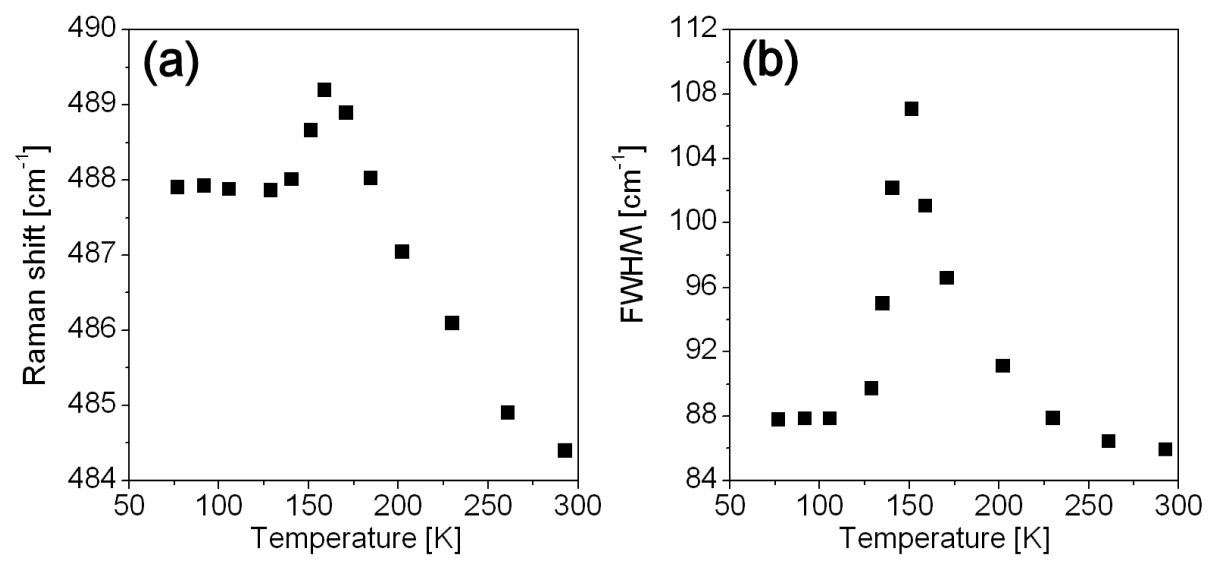

Fig. 7. Temperature dependence of the Raman shift (a) and the full width at half maximum (FWHM) (b) of the translation mode $\left(484 \mathrm{~cm}^{-1}\right)$ for SLG crystals. 
Raman spectroscopy is one of the methods permitting characterization of the SLG oxide crystals. The temperature dependences of the Raman shift and FWHM of the phonon bands obtained from the Raman scattering spectra revealed discontinuities near $150 \mathrm{~K}$ - Fig. 7. The discontinuity of the SLG translation mode at $484 \mathrm{~cm}^{-1}$ of SLG is related to the deformation of the oxygen octahedral $\mathrm{GaO}_{4}$. This discontinuity is interpreted as evidence of the deformation of oxygen atoms surrounding the Ga ion [17-19]. Two types of oxide defects are possible in these crystals, related either to the excess of oxygen built into interstitial positions or to the deficiency of oxygen leading to the appearance of oxygen gaps. The interstitial positions oxygen atoms were determined by the tight-binding linear muffin-tin orbital (LMTO) as $(0.5,0,-0.2361)$ and $(0,0.5,-0.2361)$ [18].

\section{Raman scattering in molecular crystals}

The crystal of TAHSe has been a subject of intense study undertaken to describe its crystalline phases and mechanisms of its phase transitions, which have not been resolved yet. The Raman scattering study was undertaken to explain the molecular mechanisms of the phase transitions, the role of the molecular groups ordering and ordering of protons in the hydrogen bonds.

Temperature investigation of the Raman scattering permits a direct observation of changes in the lattice dynamics related to the phase transitions. The molecular TAHSe crystals undergo 5 phase transitions and six structural phases, shown in the scheme below

$$
\frac{R \overline{3} m}{(\mathrm{I})} \frac{\rightarrow}{330 \mathrm{~K}} \frac{R \overline{3}}{(\mathrm{II})} \underset{298 \mathrm{~K}}{\rightarrow} \frac{P \overline{1}}{(\mathrm{III})} \underset{273 \mathrm{~K}}{\rightarrow} \frac{C 2 / c}{(\mathrm{IV})} \underset{181 \mathrm{~K}}{\rightarrow} \frac{C c}{(\mathrm{~V})} \underset{101 \mathrm{~K}}{\rightarrow} \frac{C c}{(\mathrm{VI})}
$$

Two of these phase transitions, those occurring at $181 \mathrm{~K}$ and $101 \mathrm{~K}$, are ferroelectric.

The transition to the ferroelectric phase $\mathrm{V}$ is accompanied by an increase in the number of the Raman bands to nine. Four of them correspond to the translational and librational vibrations of the $\mathrm{SO}_{4}$ group, while the other five come from the translational and librational vibrations of the $\mathrm{NH}_{4}$ groups. Two types of $\mathrm{NH}_{4}^{+}$ ions of different activation energies $(21.1 \mathrm{~kJ} / \mathrm{mol}, 6.2 \mathrm{~kJ} / \mathrm{mol})$ perform isotropic rotation [20]. The inhibition of the rotation of the higher activation energy is manifested in the Raman spectrum by the librational modes. Six modes were recorded in the range of bending vibrations of the $\mathrm{SO}_{4}$ groups, while eleven ones - in the range of the stretching vibrations. An increase in the number of modes following from the separation of modes in the range of internal vibrations testifies to the ordering of protons in the hydrogen bonds of dimers and the orientational ordering of the $\mathrm{SO}_{4}$ groups. Figure 8 presents the temperature dependences of the wave numbers for the bending and stretching vibrations of the $\mathrm{SeO}_{4}$ groups and librational vibrations of the $\mathrm{NH}_{4}$ groups.

The transition at $101 \mathrm{~K}$ to phase VI causes a further increase in the crystal ordering manifested by an increase in the number of the Raman bands. The 


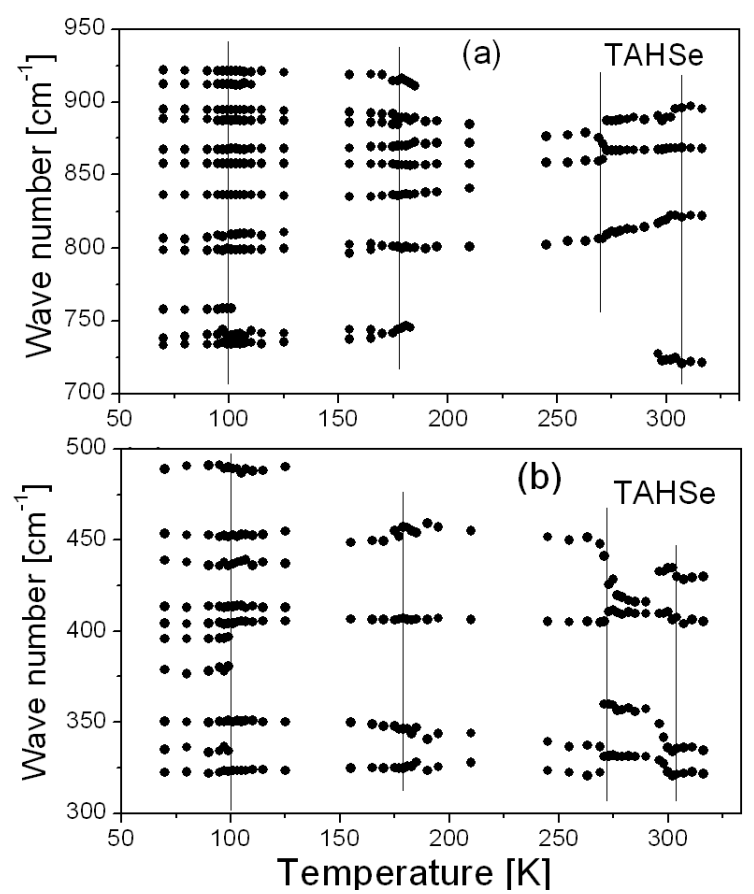

Fig. 8. The dependence of the wave number of the Raman bands for the bending vibrations of the $\mathrm{SeO}_{4}$ group and librations of $\mathrm{NH}_{4}$ group (a) and stretching vibrations of $\mathrm{SeO}_{4}$ group (b) for the TAHSe crystals.

number of vibrations is assigned to the librations of the $\mathrm{NH}_{4}$ groups of the $\mathrm{NH}_{4}^{+}$ ions of the second type possessing the lower energy of activation. This fact testifies to the total ordering of the crystal structure of the TAHSe crystal. After the phase transition the number of vibrations observed in the range of the bending and stretching bands of the $\mathrm{SO}_{4}$ group also increases. The reason for the separation of the Raman bands in the spectrum is the proton ordering in the hydrogen bonds and in consequence the formation of inequivalent $\mathrm{SO}_{4}$ groups. Literature proposes a model of the ferroelectric phase transitions in the TAHSe crystal according to which the ferroelectric properties of phase $\mathrm{V}$ are the result of the orientational ordering of the groups $\mathrm{NH}_{4}$ and $\mathrm{SO}_{4}$. Our results of the Raman scattering study also suggest such a model, moreover, it has been supported by the results of the other authors reporting the IR results [21, 22].

\section{Raman scattering in polymers}

\subsection{Determination of the phase transition temperatures}

Recently, the physical properties of a wide class of materials of disordered or much defected internal structure have become of increasing interest. These materials include a group of substances representing the so-called soft matter: liquids, 
gels, liquid crystals, and polymers. This section deals with the Raman scattering study of a homologue series of polyoxyethylene glycols, belonging to high-molecular systems. These polymers, known as PEG, have found a wide application in industry as surfactants, densifiers, stabilizers, and wetters. They have been also of interest in biotechnology and medicine as they can be a simple model of the diffusion molecule in proteins [23-25].

From the Raman scattering spectra of PEG recorded at room temperature (solid state) and at $343 \mathrm{~K}$ (liquid) it has been established that the shape, integral intensity and FWHM of the phonon bands depend on temperature.

Analysis of the range of frequencies, corresponding to the skeleton vibrations of a PEG molecule (Fig. 9), has shown that one of the phonon bands of the wave number $838 \mathrm{~cm}^{-1}$ appears in the spectra taken both at room temperature and at $343 \mathrm{~K}$, while the band $855 \mathrm{~cm}^{-1}$ occurs only in the spectrum recorded at room temperature and disappears at the phase transition. The band corresponding to

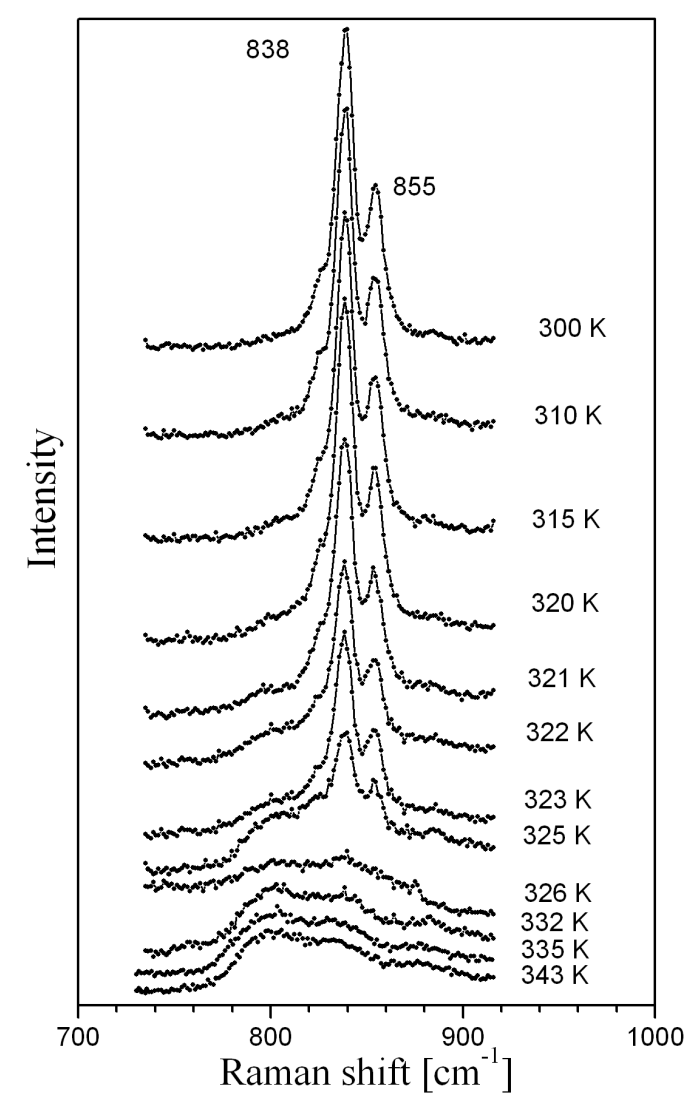

Fig. 9. The Raman bands ( 838 and $855 \mathrm{~cm}^{-1}$ ) for PEG 2000 obtained at different temperatures [28]. 

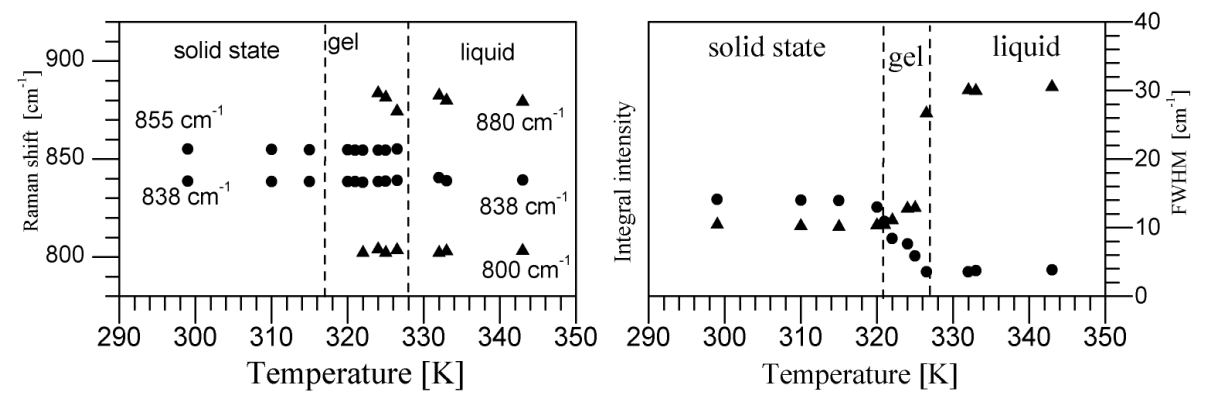

Fig. 10. Temperature dependence of the Raman shift for three states of aggregation (left) and the integral intensity (right - circle) and the FWHM of phonon band (right — triangle) for PEG 2000 [28].

the skeleton vibrations at $838 \mathrm{~cm}^{-1}$ appears in the spectra recorded for PEG in all three phases, so it was chosen for further analysis. On the basis of the temperature changes in the integral intensity and FWHM of this band (Fig. 10) it was possible to determine the temperature ranges of the phase transitions solid state-gel-liquid for PEG of different molecular weight.

\subsection{Determination of molecular ordering}

Knowing the distribution function $f(\beta)$ it is possible to conclude about the far-range orientational ordering in a given medium. The distribution function $f(\beta)$ can be expressed in a good approximation by the Legendre polynomials $P_{2}(\cos \beta)$ and $P_{4}(\cos \beta)[26]$ :

$$
f_{4}(\beta)=\frac{1}{2}+\frac{5}{2}\left\langle P_{2}\right\rangle P_{2}(\cos \beta)+\frac{9}{2}\left\langle P_{4}\right\rangle P_{4}(\cos \beta) .
$$

The Legendre polynomials $P_{2}(\cos \beta)$ and $P_{4}(\cos \beta)$ can be expressed in terms of the angle $\beta$, describing the mutual positions of monomers relative to the direction of the ordering as follows [27]:

$$
\begin{aligned}
& P_{2}(\cos \beta)=\frac{3}{2} \cos ^{2} \beta-\frac{1}{2}, \\
& P_{4}(\cos \beta)=\frac{35}{8} \cos ^{4} \beta-\frac{30}{8} \cos ^{2} \beta+\frac{5}{8} .
\end{aligned}
$$

The direction of the ordering is determined by the long axes of the polymer chains, stretched under the effect of water and ordered by the gravitational field. The degree of the molecular ordering corresponds to the mean value of the Legendre polynomial $\left\langle P_{2}\right\rangle$. The mean value of the Legendre polynomial $\left\langle P_{4}\right\rangle$ provides additional information correcting the distribution function $f(\beta)$.

The two parameters $\left\langle P_{2}\right\rangle$ and $\left\langle P_{4}\right\rangle$ can be determined from the Raman scattering spectrum. The values of these parameters can be found from the depolarization coefficients of the scattered light obtained as the ratios of certain integral intensity components $I_{z y} / I_{z z}$ and $I_{y z} / I_{y y}$, respectively. Figure 11 presents the order parameters $\left\langle P_{2}\right\rangle$ and $\left\langle P_{4}\right\rangle$ as a function of concentration of the water solution 


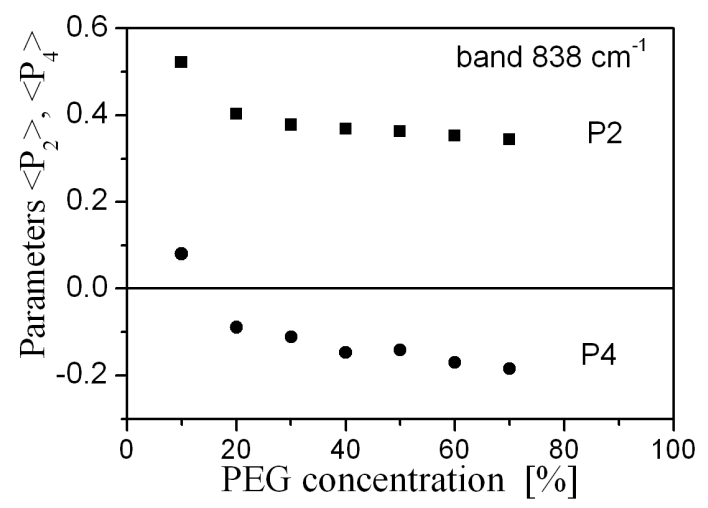

Fig. 11. The order parameters $\left\langle P_{2}\right\rangle$ (square) and $\left\langle P_{4}\right\rangle$ (circle) as a function for different PEG 1500 water solution concentrations [28].

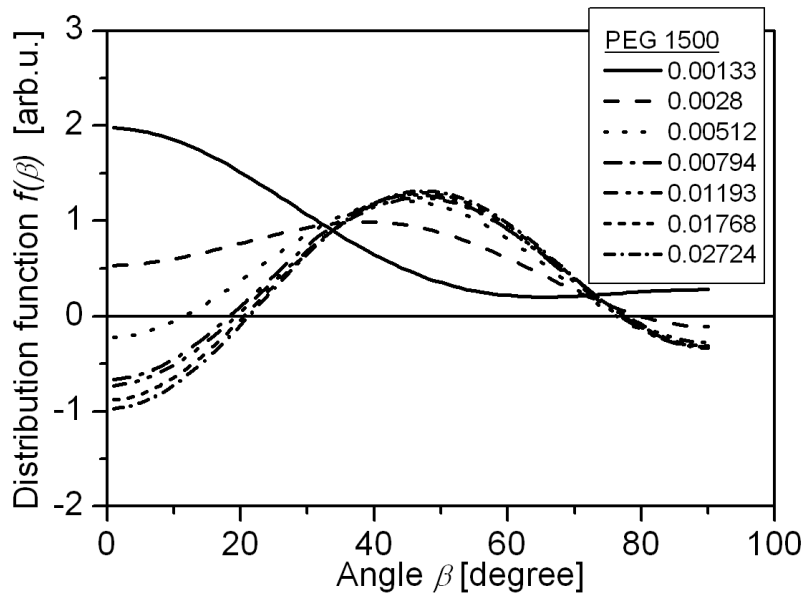

Fig. 12. The molecular distribution function $f(\beta)$ for different PEG 1500 water solution concentrations.

of PEG 1500 [28]. Figure 12 presents the distribution function $f(\beta)$ for different concentrations of the water solution of PEG 1500. Analysis of the courses of $f(\beta)$ shows that in solutions of concentrations of up to $20 \%$ the monomers in the polymer chain tend to an almost parallel arrangement. In the solutions of higher concentrations the monomers tend to the arrangement in which they are inclined at angles greater than zero. The presence of such conformations of the polymer chain can be explained by the interaction of the solvent on the polymer in the process of dissolution. This interaction counteracts the van der Waals forces between particular chains and chain segments responsible for the chain folding into random coils. In a good PEG solvent such as water the polymer random coils are subjected to strong stretching and tend to almost parallel arrangement. This conclusion is consistent with literature data [29]. 


\subsection{Conformation of the polymer chain}

Analysis of the conformational changes of the polymer chain permits a description of the structure of the PEG chains and their behaviour under the effect of external factors. In polymer chains changes in the conformation of a large number of bonds in the chain leads to the folding or unfolding of the chain. Because of the dominant contribution of carbon atoms in the PEG chain, the covalent bonds play the dominant role. Individual atoms occupy a stable spatial position determined by the angles and length of interatomic bonds. Thermal mobility of the atoms in the mer chain is manifested by rotation of the atoms of the side groups about the single bonds of the main chain. The most stable positions of the side groups are those at which the potential internal energy of the mer group reaches minimum.

It is known from literature that in the Raman scattering spectra the PEGs in the conformation gauche about the $\mathrm{C}-\mathrm{C}$ bond are assigned to the band at $1275 \mathrm{~cm}^{-1}$, while the PEGs in the conformation trans about the $\mathrm{C}-\mathrm{C}$ bond to the band at $1227 \mathrm{~cm}^{-1}[23]$. On the other hand, in the Raman spectra the PEGs in the conformation gauche about the $\mathrm{C}-\mathrm{O}$ bond are manifested by changes in the integral intensity of the band at $1136 \mathrm{~cm}^{-1}$, while the PEGs in the conformation trans about the $\mathrm{C}-\mathrm{O}$ bond are manifested by changes in the integral intensity of the band at $1118 \mathrm{~cm}^{-1}$ vs. the molecular fraction of PEG. The bands assigned to the $\mathrm{C}-\mathrm{C}$ bonds are related to the wagging vibrations of the $\mathrm{CH}_{2}$ group, while those assigned to the $\mathrm{C}-\mathrm{O}$ bonds to the twisting vibrations of the $\mathrm{CH}_{2}$ group.

Figure 13 presents the $I_{\text {gauche }} / I_{\text {trans }}$, ratio of the integral intensities of the Raman bands on the molar fraction of the water solution of PEG 1500. The ratio corresponds to the abundance of the populations in the gauche and trans conformations, assigned to the vibrations of $\mathrm{C}-\mathrm{C}$ and $\mathrm{C}-\mathrm{O}$ bonds in the polymer chain studied. As follows from the figure with increasing content of PEG in the water solution the ratio of the integral intensities $I_{\text {gauche }} / I_{\text {trans }}$ of the Raman bands assigned to the $\mathrm{C}-\mathrm{C}$ bonds decreases to unity. It means that near these

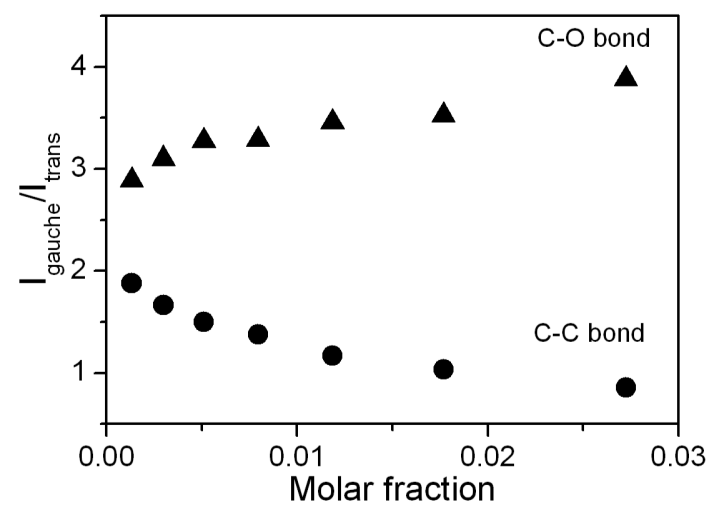

Fig. 13. Intensity ratio $I_{\text {gauche }} / I_{\text {trans }}$ for $\mathrm{C}-\mathrm{C}$ and $\mathrm{C}-\mathrm{O}$ bands as a function of mole fraction. 
bonds an equilibrium between the gauche and trans conformations is settled. On the other hand, with increasing content of PEG the ratio $I_{\text {gauche }} / I_{\text {trans }}$ assigned to the $\mathrm{C}-\mathrm{O}$ bonds increases, which means that near these bonds the population gauche dominates over the trans one. With the increase of content of water in the PEG solution the amount of the population gauche near the $\mathrm{C}-\mathrm{C}$ bonds increases, while near the $\mathrm{C}-\mathrm{O}$ bonds the amount of the population trans increases. The dominance of PEGs in the gauche conformation near $\mathrm{C}-\mathrm{C}$ bond favours the formation of hydrogen bonds joining the oxygen atoms of the polymer chain with hydrogen atoms from water. On the basis of the conformation analysis it can be concluded that in the polymer chain every second or third mer assumes the $\mathrm{C}-\mathrm{C}$ trans conformation, while the others have gauche conformation. This arrangement favours the formation of the helical structure [23, 30, 31].

As follows from analysis of Fig. 14, the ratio of the integral intensities $I_{\text {gauche }} / I_{\text {trans }}$ of the Raman bands assigned to the bonds $\mathrm{C}-\mathrm{C}$ for PEG of molecular weight below 1000 slightly increases with increasing molecular weight, which indicates that the population of the gauche conformation in a polymer chain has increased. For the polymers that are solid at room temperature (molecular weight above 1000 ), the ratio $I_{\text {gauche }} / I_{\text {trans }}$ of the Raman bands slightly decreases, which testifies to a small increase in the population of the trans conformation in the chain. The value of $I_{\text {gauche }} / I_{\text {trans }}$ lower than 1 testifies to the predominance of [CO] in the trans conformation about the $\mathrm{C}-\mathrm{C}$ bonds and hence, along the whole skeleton of the polymer chain.

Figure 14 presents the ratio $I_{\text {gauche }} / I_{\text {trans }}$ of the Raman bands assigned to the vibrations of the $\mathrm{C}-\mathrm{O}$ bond as a function of the molecular weight of a given PEG. For the liquid polymers the ratio $I_{\text {gauche }} / I_{\text {trans }}$ decreases with increasing molecular weight. For PEGs of the molecular weight above 1000, the ratio $I_{\text {gauche }} / I_{\text {trans }}$ is constant. The $I_{\text {gauche }} / I_{\text {trans }}$ changes, presented in Fig. 14, assigned to the $\mathrm{C}-\mathrm{O}$ bonds indicate the prevalence of the gauche conformation.

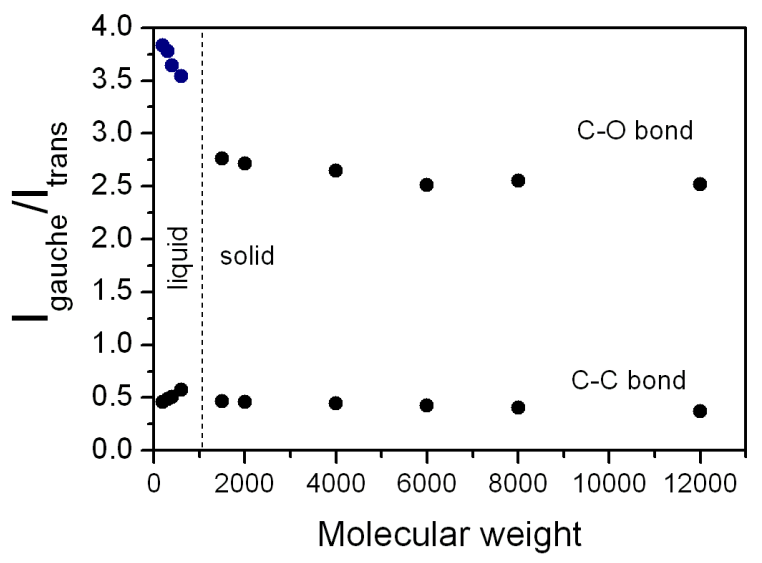

Fig. 14. Intensity ratio $I_{\text {gauche }} / I_{\text {trans }}$ for $\mathrm{C}-\mathrm{C}$ and $\mathrm{C}-\mathrm{O}$ bands as a function of average molecular weight of PEG. 
A constant value of the ratio $I_{\text {gauche }} / I_{\text {trans }}$ testifies to a stable structure of PEGs of molecular weight above 1000 .

The changes in the $I_{\text {gauche }} / I_{\text {trans }}$ ratio presented in Fig. 14 point to the dominance of the trans conformation near the $\mathrm{C}-\mathrm{C}$ bonds and the dominance of the gauche conformation near the $\mathrm{C}-\mathrm{O}$ bonds. The dominance of the trans conformation along the main skeleton of the polymer chain and of the gauche conformation near the $\mathrm{C}-\mathrm{O}$ bonds favours the formation of random coil structure. Small changes in $I_{\text {gauche }} / I_{\text {trans }}$ for the liquid polymers at room temperature (molecular weight below 1000) can be a result of small reorientations of these chains within the random coil [32].

\section{Summary}

The paper presents the possible applications of the Raman scattering data for characterization of different materials. On the basis of the frequencies of the phonon bands and the plasmon damping constant determined from the Raman scattering spectra, a mechanism of scattering of free charge carriers in the ZnSe crystals has been proposed. It has been shown that analysis of the Raman scattering spectra also enables a determination of concentration of free charge radicals, elasticity constants, and the internal stress parameter in the ZnSe crystals. Another piece of information obtained from the Raman spectra is the range of splitting of the optical modes TO-LO: (ZnSe)-like, (MgSe)-like, and (BeSe)-like in the diphase crystals $\mathrm{ZnMg} / \mathrm{BeSe}$. On the basis of the frequency of the local mode and the internal mode and the optical frequencies of the transversal and longitudinal modes found from the Raman spectrum it has been possible to determine the force constants of the interactions between particular atoms. It has been also established that the ionic character of the bonds in ZnSe crystals increases as a result of the replacement of the $\mathrm{Zn}$ atoms by $\mathrm{Mg}$ or Be ones.

Two types of oxygen defects have been found possible in the SLG crystals, either related to the oxygen deficiency (oxygen gaps) or to its excess, when oxygen is built-in in the interstitial sites. The possible positions of these defects in the SLG crystal structure have been indicated.

Raman scattering spectra of TAHSe crystals have provided the information that the ferroelectric properties of phase $\mathrm{V}$ are a result of the orientational ordering of the $\mathrm{NH}_{4}$ and $\mathrm{SO}_{4}$ groups. A transition to phase VI is caused by a further ordering in the crystal, which is evidenced by an increase in the number of the librational vibrations of the $\mathrm{NH}_{4}$ groups proving the complete ordering of the structure of the TAHSe crystal.

Analysis of the temperature variations in the integral intensity and FWHM of the phonon bands has permitted determination of the temperature ranges of the phase transitions solid state-gel-liquid in PEGs of different molecular weights. The Raman scattering spectra taken for water solutions of PEGs permitted determination of the order parameters dependent on the scattered light depolarization coefficients and the function of molecular distribution for different PEG concentra- 
tions. It has been shown that in water solutions of PEGs at a concentration below $20 \%$, the monomers in the polymer chain tend to an almost parallel arrangement. In solutions of higher PEG concentrations the monomers tend to assume positions at a certain angle to one another. Such behaviour can be explained by the solvent effect on the polymer in the process of dissolution. In a good solvent such as, e.g. water, statistical coils are subjected to strong stretching as the chains tend to an almost parallel mutual arrangement. With an increase ion contribution of water in the PEG solutions both the population of the mers in the gauche conformation about the $\mathrm{C}-\mathrm{C}$ bonds and the mers in the trans conformation about the $\mathrm{C}-\mathrm{O}$ bonds increase. The presence of the mers in the gauche conformation about the $\mathrm{C}-\mathrm{C}$ bond favours the formation of hydrogen bonds in the polymer chain. The structure of the polymer chain in which every second or third mer assumes the $\mathrm{C}-\mathrm{C}$ trans conformation and the others assume the gauche conformation, favours the formation of helices. Changes in the value of the ratio $I_{\text {gauche }} / I_{\text {trans }}$ as a function of the molecular weight indicate the dominance of the mers in the trans conformation about the $\mathrm{C}-\mathrm{C}$ bond and the gauche conformation about the $\mathrm{C}-\mathrm{O}$ bonds. The dominance of the trans conformation along the main skeleton of the polymer chain and the gauche conformation about the $\mathrm{C}-\mathrm{O}$ bond favours the formation of statistical coils of molecules.

The results discussed in the paper have illustrated the usefulness of the Raman scattering study for characterization of physical properties of different materials. The results have been in agreement with the data obtained by other methods.

\section{Acknowledgments}

This work was supported in part by the Research Project PB 64-022/BW/2006 of Poznań University of Technology. The author wishes to thank Dr. eng. M. Mühle, Dr. T. Runka and Dr. M. Szybowicz from the Faculty of Technical Physics, Poznań University of Technology for experimental work and Prof. Z. Błaszczak from the Faculty of Physics, Adam Mickiewicz University for discussions.

\section{References}

[1] M.V. Klein, B.N. Ganguly, P.J. Colwell, Phys. Rev. B 6, 2380 (1973).

[2] D.T. Hon, W.L. Faust, Appl. Phys. 1, 24 (1973).

[3] H. Yugami, S. Nakashima, K. Sakai, H. Kojima, M. Hangyo, A. Mitsuishi, J. Phys. Soc. Jpn. 56, 1881 (1987).

[4] M. Kozielski, M. Drozdowski, W. Bała, F. Firszt, Acta Phys. Pol. A 73, 369 (1988).

[5] W. Bała, F. Firszt, M. Grinberg, M. Drozdowski, M. Kozielski, Acta Phys. Pol. A 75, 75 (1989).

[6] M. Cardona, in: Light Scattering in Solids II, Eds. M. Cardona, G. Güntherodt, Springer, Berlin 1982, p. 19.

[7] P.N. Kaeating, J. Appl. Phys. 33, 3371 (1962). 
[8] K. Kunc, M. Balkanski, M.A. Nusimovici, Phys. Status Solidi B 71, 341 (1975); Phys. Status Solidi B 72, 229 (1975); Phys. Status Solidi B 72, 249 (1975).

[9] M.R. Martin, Phys. Rev. B 1, 4005 (1970).

[10] A.A. Maradudin, in: Localized Excitations in Solids, Ed. R.F. Wallis, Plenum, New York 1968, p. 1.

[11] S.P.S. Porto, in: Light Scattering Spectra of Solids, Ed. G.B. Wright, Springer, Berlin 1969, p. 1.

[12] M. Kozielski, M. Szybowicz, F. Firszt, S. Łȩgowski, H. Męczyńska, B. Sekulska, J. Szatkowski, W. Paszkowicz, Acta Phys. Pol. A 90, 1040 (1996).

[13] M. Kozielski, M. Szybowicz, F. Firszt, S. Łȩgowski, H. Męczyńska, J. Szatkowski, W. Paszkowicz, Cryst. Res. Technol. 34, 699 (1999).

[14] I.F. Chang, S.S. Mitra, Phys. Rev. 172, 924 (1968).

[15] L. Peterson, A. Petrou, W. Giriat, A. Ramdas, S. Rodriguez, Phys. Rev. B 33, 1160 (1986).

[16] W. Ryba-Romanowski, S. Gołąb, I. Sokólska, W.A. Pisarski, G. Dominiak-Dzik, A. Pajączkowska, M. Berkowski, J. Alloys. Comp. 217, 263 (1995).

[17] M. Drozdowski, M. Kozielski, A. Pajączkowska, Acta Phys. Pol. A 92, 139 (1997).

[18] M. Kozielski, A. Jezierski, D. Kasprowicz, M. Szybowicz, Cryst. Res. Technol. 34, 715 (1999).

[19] A. Pajạczkowska, A. Gloubokov, Prog. Crystal Growth Charact. 36, 123 (1998).

[20] J. Tritt-Goc, N. Piślewski, S.K. Hoffmann, M. Augustyniak, Phys. Status Solidi B 176, K13 (1993).

[21] G. Raimbault, F. Romain, A. Lautie, J. Raman Spectrosc. 23, 147 (1992).

[22] M. Kamoun, M. Halouani, A. Daoud, Phase Transit. 9, 327 (1987).

[23] R. Begum, H. Matsuura, J. Chem. Soc. Faraday Trans. 93, 3839 (1997).

[24] Y. Nakajima, H. Yoshida, S. Kobinata, S. Maeda, J. Phys. Soc. Jpn. 49, 1140 (1980).

[25] W. Przygocki, A. Włochowicz, Polymer Physics (Fizyka polimerów), Wydawnictwo Naukowe PWN SA, Warszawa 2001 (in Polish).

[26] L.G.P. Dalmolen, S.J. Picken, A.F. de Jong, W.H. de Jeu, J. Phys. (France) 46, 1443 (1985).

[27] M. Kozielski, D. Bauman, M. Drozdowski, Z. Salomon, Mol. Cryst. Liq. Cryst. 142, 1 (1987).

[28] M. Kozielski, M. Mühle, Z. Błaszczak, J. Mol. Liquids 111, 1 (2004).

[29] B. Heymann, H. Grubmüller, Chem. Phys. Lett. 307, 425 (1999).

[30] W.N. Cwietkow, W.J. Eskin, S.J. Frenkel, Macromolecule Structure in Solutions (Struktura makroczastek w roztworach), WNT, Warszawa 1968 (in Polish).

[31] K. Tasaki, J. Am. Chem. Soc. 118, 8459 (1996).

[32] M. Mühle, Ph.D. Thesis, Poznań University of Technology, Poznań 2005 (in Polish). 